\title{
Avoid the quasi-equilibrium assumption when evaluating the electrocatalytic oxygen evolution reaction mechanism by Tafel slope analysis
}

\author{
Aaron T. Marshall*, Laurent Vaisson-Béthune \\ Department of Chemical and Process Engineering, MacDiarmid Institute for Advanced Materials and \\ Nanotechnology, University of Canterbury, Christchurch, New Zealand
}

\begin{abstract}
Tafel slope analysis is a powerful tool in comparing experimental data to a proposed reaction mechanism. Typically, in order to simplify the analysis, the non-rate-determining steps in the reaction mechanism are assumed to be in quasi-equilibrium. Here, Tafel analysis of the oxygen evolution reaction following the electrochemical oxide mechanism is performed using a full kinetic model. It is shown that this model (which uses the steady-state assumption) predicts a larger number of Tafel slopes than if the quasi-equilibrium assumption is used, and provides predicts the surface coverages which underpin these Tafel slopes. Importantly, this model predicts Tafel slopes of 30, 40, 60 and $120 \mathrm{mV}$, all of which are experimentally found on $\mathrm{IrO}_{2}$ and $\mathrm{RuO}_{2}$ anodes. Models using the quasi-equilibrium assumption fail to predict some Tafel regions, as these can occur when a non-rate-determining step is not at quasi-equilibrium.
\end{abstract}

Keywords:

Oxygen evolution reaction, Tafel slope, Reaction mechanism, Quasi-equilibrium, Electrocatalysis

\footnotetext{
*Corresponding author

Email address: aaron.marshall@canterbury.ac.nz (Aaron T. Marshall)
} 


\section{Introduction}

The oxygen evolution reaction (OER) is one of the most important electrode reactions [1]. Unfortunately this reaction has rather poor kinetics and so large overpotentials are required to drive the OER at an industrially relevant rate. Furthermore, despite being a relatively simple reaction, the reaction mechanism is still poorly understood, and thus the development of oxygen evolution electrocatalysts is still largely trial and error based.

In order to assess the activity of electrocatalysts, the most measure the Tafel slope, exchange current, and the electrochemically active surface area. Of these, the Tafel slope has the advantage of being an intensive property of the electrocatalyst $[2,3]$ and can be an indicator of the reaction mechanism or the rate-determining step (rds). An equivalent experimentally measurable quantity which can be used in place of the classical Tafel slope is the transfer coefficient as defined by $\alpha=\left(\frac{R T}{F}\right) \frac{d \ln i}{d E}[4,5]$. For OER electrocatalysts such as $\mathrm{RuO}_{2}$ or $\mathrm{IrO}_{2}$, the Tafel slope is normally between 30 and $60 \mathrm{mV}$, but can exhibit Tafel slopes of up to $120 \mathrm{mV}$ at high overpotentials [6-11].

There are many proposed reaction mechanisms for the OER and in early work, Bockris [12] and Riddiford [13] analysed several of these and provided the Tafel slopes which should be expected if the non-rds of the reaction are at quasi-equilibrium (e.g. the electrochemical oxide mechanism can produce Tafel slopes of 15, 40 and $120 \mathrm{mV}$ ). The advantage of using the quasi-equilibrium assumption is that the kinetic parameters do not need to be known in order to predict the Tafel slope, although recently others have suggested that the steady-state approximation may be more useful [14]. Others have also highlighted that the quasi-equilibrium assumption may over simplify the kinetic analysis of multi-step reactions $[15,16]$. Despite the limitations in using the quasi-equilibrium assumption, many have used this approach and ruled out or modified mechanisms on the basis that measured Tafel slopes do not match the predicted values. For example, the electrochemical oxide mechanism has been modified to include an additional step on the basis of finding Tafel slopes of $60 \mathrm{mV}$ on $\mathrm{IrO}_{2}$ based electrodes $[6,10,17]$.

In this paper we show that the electrochemical oxide mechanism is capable of producing Tafel slopes other than those predicted by the quasi-equilibrium assumption approach, suggesting that Tafel slope analysis to evaluate a reaction mechanism should avoid the quasi-equilibrium assumption.

\section{Mathematical Model}

The OER proceeds by the electrochemical oxide mechanism via three consecutive steps [12]:

$$
\begin{gathered}
\mathrm{S}+\mathrm{H}_{2} \mathrm{O} \rightleftharpoons \mathrm{S}-\mathrm{OH}+\mathrm{H}^{+}+\mathrm{e}^{-} \\
\mathrm{S}-\mathrm{OH} \rightleftharpoons \mathrm{S}-\mathrm{O}+\mathrm{H}^{+}+\mathrm{e}^{-} \\
\mathrm{S}-\mathrm{O} \rightleftharpoons \mathrm{S}+\frac{1}{2} \mathrm{O}_{2}
\end{gathered}
$$

where $\mathrm{S}$ is a free surface site. The reaction rates for each step are given by:

$$
r_{1}=k_{1} C_{\mathrm{H}_{2} \mathrm{O}} N_{t o t}\left(1-\theta_{\mathrm{OH}}-\theta_{\mathrm{O}}\right)-k_{-1} C_{\mathrm{H}^{+}} N_{t o t} \theta_{\mathrm{OH}}
$$




$$
\begin{gathered}
r_{2}=k_{2} N_{t o t} \theta_{\mathrm{OH}}-k_{-2} C_{\mathrm{H}^{+}} N_{t o t} \theta_{\mathrm{O}} \\
\left.r_{3}=k_{3} N_{t o t} \theta_{\mathrm{O}}-k_{-3} C_{\mathrm{O}_{2}}^{1 / 2} N_{t o t}\left(1-\theta_{\mathrm{OH}}-\theta_{\mathrm{OH}}\right)\right)
\end{gathered}
$$

where $k_{1}$ and $k_{-1}$ etc. are forward and back rate constants, $C$ are concentrations and $N_{t o t}$ is the number of surface sites per unit electrode area. $\theta_{\mathrm{OH}}$ and $\theta_{\mathrm{O}}$ are the surface coverage of $\mathrm{OH}$ and $\mathrm{O}$ respectively, with the fraction of free sites given by $1-\theta_{\mathrm{OH}}-\theta_{\mathrm{OH}}$.

The rate constants for reactions 1 and 2 are assumed to be potential dependent as per Butler-Volmer kinetics, e.g.:

$$
\begin{gathered}
k_{i}=k_{i}^{0} \exp ^{\frac{\beta F}{R T}\left(E-E_{r e v}\right)} \\
k_{-i}=k_{-i}^{0} \exp ^{\frac{-(1-\beta) F}{R T}\left(E-E_{r e v}\right)}
\end{gathered}
$$

where, $E$ and $E_{r e v}$ are the anode and reversible potential for the OER respectively and $\beta, F, R$ and $T$ are the symmetry factor, Faraday constant, gas constant and temperature respectively. Here it is assumed that $\beta=0.5$ to facilitate the direct comparison of the Tafel slopes calculated here to those reported for the same mechanism when the quasiequilibrium assumption has been used. However is likely that $\beta$ can deviate considerable from 0.5 for processes involving adsorbed species and thus comparison of mechanisms with experimental data should take this into consideration [18].

\subsection{Equilibrium constraints}

The thermodynamics of the overall reaction requires that:

$$
K=\frac{C_{\mathrm{H}^{+}}^{2} C_{\mathrm{O}_{2}}^{1 / 2}}{C_{\mathrm{H}_{2} \mathrm{O}}}=K_{1} K_{2} K_{3}
$$

where $K$ is the overall equilibrium constant for the reaction and $K_{1}, K_{2}$ and $K_{3}$ are the individual equilibrium constants for each reaction step and where $K_{1}=k_{1}^{0} / k_{-1}^{0}$, $K_{2}=k_{2}^{0} / k_{-2}^{0}$ and $K_{3}=k_{3} / k_{-3}$.

At the reversible potential (i.e. $E-E_{\text {rev }}=0$ ), all three reaction steps must be in equilibrium and thus:

$$
\theta_{\mathrm{OH}, \text { rev }}=\frac{K_{1}}{\frac{C_{\mathrm{H}^{+}}}{C_{\mathrm{H}_{2} \mathrm{O}}}+K_{1}}\left(1-\theta_{\mathrm{O}, \text { rev }}\right)=\frac{C_{\mathrm{H}^{+}} \theta_{\mathrm{O}, \text { rev }}}{K_{2}}=-\frac{K_{3}}{C_{\mathrm{O}_{2}}^{1 / 2}} \theta_{\mathrm{O}, \text { rev }}-\theta_{\mathrm{O}, \text { rev }}+1
$$

This enables the surface coverages at the reversible potential to be calculated from two of the equilibrium constants. In this case using $K_{2}$ and $K_{3}$ gives:

$$
\theta_{\mathrm{O}, \text { rev }}=\frac{1}{1+\frac{K_{3}}{C_{\mathrm{O}_{2}}^{1 / 2}}+\frac{C_{\mathrm{H}^{+}}}{K_{2}}}
$$




\subsection{Steady state oxygen evolution}

During steady-state oxygen evolution, $\theta_{\mathrm{OH}}$ and $\theta_{\mathrm{O}}$ will be at steady-state:

$$
\begin{gathered}
\frac{d \theta_{\mathrm{OH}}}{d t}=k_{1} C_{\mathrm{H}_{2} \mathrm{O}}\left(1-\theta_{\mathrm{OH}}-\theta_{\mathrm{O}}\right)-k_{-1} C_{\mathrm{H}^{+}} \theta_{\mathrm{OH}}-k_{2} \theta_{\mathrm{OH}}+k_{-2} C_{\mathrm{H}^{+}} \theta_{\mathrm{O}}=0 \\
\frac{d \theta_{\mathrm{O}}}{d t}=k_{2} \theta_{\mathrm{OH}}-k_{-2} C_{\mathrm{H}^{+}} \theta_{\mathrm{O}}-k_{3} \theta_{\mathrm{O}}+k_{-3} C_{\mathrm{O}_{2}}^{1 / 2}\left(1-\theta_{\mathrm{OH}}-\theta_{\mathrm{O}}\right)=0
\end{gathered}
$$

from which $\theta_{\mathrm{OH}}$ and $\theta_{\mathrm{O}}$ to be determined as a function of potential:

$$
\begin{gathered}
\theta_{\mathrm{OH}}=\frac{k_{-3} C_{\mathrm{O}_{2}}^{1 / 2} k_{-2} C_{\mathrm{H}^{+}}+k_{1} C_{\mathrm{H}_{2} \mathrm{O}}\left(k_{3}+k_{-2} C_{\mathrm{H}^{+}}\right)}{\left(k_{2}+k_{1} C_{\mathrm{H}_{2} \mathrm{O}}+k_{-1} C_{\mathrm{H}^{+}}\right)\left(k_{3}+k_{-2} C_{\mathrm{H}^{+}}+k_{-3} C_{\mathrm{O}_{2}}^{1 / 2}\right)-\left(k_{2}-k_{-3} C_{\mathrm{O}_{2}}^{1 / 2}\right)\left(k_{-2} C_{\mathrm{H}^{+}}-k_{1} C_{\mathrm{H}_{2} \mathrm{O}}\right)} \\
\theta_{\mathrm{O}}=\frac{\theta_{\mathrm{OH}}\left(k_{2}+k_{1} C_{\mathrm{H}_{2} \mathrm{O}}+k_{-1} C_{\mathrm{H}^{+}}\right)-k_{1} C_{\mathrm{H}_{2} \mathrm{O}}}{\left(k_{-2} C_{\mathrm{H}^{+}}-k_{1} C_{\mathrm{H}_{2} \mathrm{O}}\right.}
\end{gathered}
$$

Finally, from equations 1, 2, 4, 5, 11 and 12, the current density can be calculated as a function of overpotential with:

$$
i=F\left(r_{1}+r_{2}\right)
$$

Thus by choosing values for the freely definable equilibrium constants and rate constants, steady-state polarisation curves can be easily calculated. In this paper, to enable direct comparison of the simulated polarisation curves, the current density is normalised by the exchange current density $\left(i_{0}\right)$, obtained by extrapolating the Tafel line to the reversible potential. It should be pointed out that due to the stoichiometry of the OER, the measured $i-E$ behaviour at the reversible limit maybe more complex if obtained by potentiodynamic techniques [19].

\subsection{Rate-determining step and validity of the quasi-equilibrium assumption}

Using this model, the rds for any combination of rate and equilibrium constants can be easily determined. While the net rate of each step will be the same during steadystate oxygen evolution, the forward rate component of each step will be different, the slowest of which corresponds to the rds. These are calculated by:

$$
\begin{gathered}
\overrightarrow{r_{1}}=k_{1} C_{\mathrm{H}_{2} \mathrm{O}} N_{t o t}\left(1-\theta_{\mathrm{OH}}-\theta_{\mathrm{O}}\right) \\
\overrightarrow{r_{2}}=k_{2} N_{t o t} \theta_{\mathrm{OH}} \\
\overrightarrow{r_{3}}=k_{3} N_{t o t} \theta_{\mathrm{O}}
\end{gathered}
$$

It is also possible to evaluate whether the a non-rds is close to quasi-equilibrium, by comparing the forward rate components of these steps with the net rate. If a forward rate component is many orders of magnitude higher than the net rate, the forward rate component will be approximately equal to the backward rate component and thus the step can be assumed to be in quasi-equilibrium. 


\section{Results and Discussion}

By selecting appropriate equilibrium and rate constants, polarisation curves with Tafel slopes of $120 \mathrm{mV}$ or dual Tafel slopes of 40 and $120 \mathrm{mV}$ can obtained (Figure 1). This is identical to that predicted when using the quasi-equilibrium assumption, with the Tafel slope of $120 \mathrm{mV}$ and $40 \mathrm{mV}$ occurring when step 1 and step 2 are the rds respectively $[12,13]$. It should be pointed out that Bockris [12] suggested that dual Tafel slopes of 40 and $120 \mathrm{mV}$ can arise when step 2 is the rds, whereas Riddiford [13] showed that this occurs when the rds switches from step 2 to step 1 - which is what is observed in the current simulations. It is also possible to see that the prediction of a limiting current at high overpotential (when step 3 limits and $\theta_{\mathrm{O}} \rightarrow 1$, resulting in an infinite Tafel slope) is also be obtained (Figure 1).

Both Bockris [12] and Riddiford [13] show that if step 3 is the rds and if one assumes that $\theta_{\mathrm{OH}} \rightarrow 0$ and if steps 1 and 2 are in quasi-equilibrium, a Tafel slope of $15 \mathrm{mV}$ would be observed. However in our simulations, when the parameters are defined such that the system falls under these conditions, a Tafel slope of $15 \mathrm{mV}$ is not observed. This is because the potential range where the required conditions are meet is relatively small and thus does not result is clear Tafel behaviour (we normally consider Tafel behaviour is observed when the slope is the same for at least 1 decade in current density). Interestingly, we find no literature reporting experimental Tafel slopes of 15 $\mathrm{mV}$, which may confirm our conclusion that the conditions required to achieve this Tafel slope only occur over a small potential range.

One apparent limitation of the electrochemical oxide mechanism is that is does not give Tafel slopes of 30 and $60 \mathrm{mV}$ - the typical values observed for $\mathrm{RuO}_{2}$ and $\mathrm{IrO}_{2}$ anodes $[6-11,17]$. In fact several authors appear to rule out the electrochemical oxide mechanism on the basis that the experimental Tafel slopes do not match those predicted from the mechanism, and then develop new mechanisms, to overcome this apparent limitation $[6,10,13,17]$. However, we believe that this limitation only arises due to the use of the quasi-equilibrium assumption and is not inherent of the mechanism.

To illustrate this, polarisation curves have been calculated for a range of equilibrium constants (which reflect the relative adsorption strength of the reaction intermediates), and show that the electrochemical oxide mechanism can result in Tafel slopes of 30 and $60 \mathrm{mV}$ (Figure 2) when step 3 is the rds. These Tafel slopes are characteristic of the surface coverages, with the Tafel slope of $30 \mathrm{mV}$ occurring when $\theta_{\mathrm{OH}}$ and $\theta_{\mathrm{O}} \approx 0$ (i.e. when $K_{3}>>K_{1}$ and $K_{2}$ ) and the Tafel slope of $60 \mathrm{mV}$ occurs when $\theta_{\mathrm{O}} \approx 0$ (thereby slowing step 3 ) and $\theta_{\mathrm{OH}} \approx 1$, which slows step 1 to a comparable (albeit slightly faster) rate to step 3. Under some conditions, a dual Tafel slope of 30 and $60 \mathrm{mV}$ is also possible, when the potential dependence of the surface coverages gives $\theta_{\mathrm{OH}}$ and $\theta_{\mathrm{O}} \approx 0$ at low potentials and $\theta_{\mathrm{OH}} \approx 1$ at high potentials. Similarly, it is also possible to obtain a Tafel slope of $40 \mathrm{mV}$ (normally characteristic of step 2 limiting), when step 1 limits provided that $\theta_{\mathrm{OH}}$ is high. In all cases, a limiting current is observed when step 3 is the rds and $\theta_{\mathrm{O}} \rightarrow 1$.

To expand on this finding, polarisation curves were calculated for a wide range of equilibrium constants and analysed for their Tafel slopes and rds (Figure 3). It should be noted that qualitatively similar results are obtained if different sets of rate constants 
are used in these simulations. As discussed above, there are quite large regions where Tafel slopes of 30, 40, 60 and $120 \mathrm{mV}$ can be observed in the polarisation curves, with very clear boundaries between these regions. One interesting feature of this analysis, is the region where no clear Tafel behaviour is observed ${ }^{1}$ (central region in Figure 3A). This region corresponds to where no single step is clearly the rds and/or if none of the steps are close to quasi-equilibrium. Such polarisation curves are not typically seen in experimental literature, which can be rationalised by the fact that this region occurs when the rates for each reaction step are similar and finding an electrocatalyst which has comparable activity over a wide potential for each step in the reaction pathway seems improbable [14].

While the analysis presented here shows that the electrochemical oxide mechanism describes the typical behaviour of $\mathrm{IrO}_{2}$ and $\mathrm{RuO}_{2}$ anodes, it is not our intention to suggest that this mechanism is correct. Indeed others have suggested that the combination of adsorbed oxygen atoms to form molecular oxygen (step 3 in the discussed mechanism) appears to be less favourable than other oxygen forming steps found in other mechanisms [20-22]. Rather, we believe that this work shows that one must be careful when testing a mechanism against experimental data, especially when the quasiequilibrium assumption is used to simplify the analysis. Naturally, one outcome of the approach used here is the ease of which the model can successfully fit experimental data due to the number of freely definable kinetic parameters within the model. Thus to successfully confirm that experimental data fits a particular reaction mechanism, factors in addition to the Tafel slope should be considered (e.g. reaction order with respect to $\mathrm{H}^{+}$or measurement of surface coverages).

\section{Conclusions}

The use of the quasi-equilibrium assumption when applied to the OER following the electrochemical oxide mechanism underestimates number of unique Tafel slopes which are possible. Specifically, by using a full kinetic model of the reaction mechanism, it is shown that the electrochemical oxide mechanism is capable of producing Tafel slopes of 30,40, 60 and $120 \mathrm{mV}$, all of which cover the normal Tafel slopes found for $\mathrm{IrO}_{2}$ or $\mathrm{RuO}_{2}$ anodes. While only the electrochemical oxide pathway is considered here, the results indicate that such analysis should be performed when evaluating other reaction mechanisms rather than relying on the more straightforward quasi-equilibrium assumption.

\section{References}

[1] G. M. Whitesides, G. W. Crabtree, Don't forget long-term fundamental research in energy, Science 315 (5813) (2007) 796-798.

\footnotetext{
${ }^{1}$ It should be noted that the distinction between Tafel and non-Tafel behaviour is not necessarily clear and depends on the criteria used to determine the Tafel region. In this paper we consider Tafel behaviour is observed when the slope is the same (within $\pm 1 \mathrm{mV}$ ) for approx. 1 decade in current density
} 
[2] E. Guerrini, S. Trasatti, Recent Developments in Understanding Factors of Electrocatalysis, Russian J. Electrochem. 42 (10) (2006) 1017-1025.

[3] S. Trasatti, Electrocatalysis: understanding the success of DSA, Electrochim. Acta $45(15-16)(2000) 2377-2385$.

[4] R. Guidelli, R. G. Compton, M. Feliu Juan, E. Gileadi, J. Lipkowski, W. Schmickler, S. Trasatti, Defining the transfer coefficient in electrochemistry: An assessment (IUPAC Technical Report), Pure Appl. Chem. 86 (2) (2014) 245-258.

[5] R. Guidelli, R. G. Compton, M. Feliu Juan, E. Gileadi, J. Lipkowski, W. Schmickler, S. Trasatti, Definition of the transfer coefficient in electrochemistry (IUPAC Recommendations 2014), Pure Appl. Chem. 86 (2) (2014) 259-262.

[6] M. E. G. Lyons, S. Floquet, Mechanism of oxygen reactions at porous oxide electrodes. Part 2-Oxygen evolution at $\mathrm{RuO}_{2}, \mathrm{IrO}_{2}$ and $\mathrm{Ir}_{x} \mathrm{Ru}_{1-x} \mathrm{O}_{2}$ electrodes in aqueous acid and alkaline solution, Phys. Chem. Chem. Phys. 13 (12) (2011) 5314-5335.

[7] F. Mattos-Costa, P. de Lima-Neto, S. Machado, L. Avaca, Characterisation of surfaces modified by sol-gel $\mathrm{Ru}_{x} \mathrm{Ir}_{1-x} \mathrm{O}_{2}$ coatings for oxygen evolution in acid medium, Electrochim. Acta 44 (1998) 1515-1523.

[8] Y. Roginskaya, T. Varlamova, M. Goldshtein, T. Belova, B. Galyamov, R. Shifrina, V. Shepelin, V. Fateev, Formation, structure and electrochemical properties of $\mathrm{IrO}_{2}-\mathrm{RuO}_{2}$ oxide electrodes, Mater. Chem. Phys. 30 (1991) 101-113.

[9] R. Yeo, J.Orehotsky, W. Visscher, S. Srinivasan, Ruthenium-based mixed oxides as electrocatalysts for oxygen evolution in acid electrolytes, J. Electrochem. Soc. 128 (1981) 1900-1904.

[10] L. A. DeFaria, J. F. C. Boodts, S. Trasatti, Electrocatalytic properties of ternary oxide mixtures of composition $\mathrm{Ru}_{0.3} \mathrm{Ti}_{(0.7-x)} \mathrm{Ce}_{x} \mathrm{O}_{2}$ : Oxygen evolution from acidic solution, J. Appl. Electrochem. 26 (11) (1996) 1195-1199.

[11] C. Angelinetta, S. Trasatti, L. D. Atanasoska, Z. S. Minevski, R. T. Atanasoski, Effect of preparation on the surface and electrocatalytic properties of $\mathrm{RuO}_{2}+\mathrm{IrO}_{2}$ mixed oxide electrodes, Mater. Chem. and Phys. 22 (12) (1989) 231-247.

[12] J. O'M. Bockris, Kinetics of Activation Controlled Consecutive Electrochemical Reactions: Anodic Evolution of Oxygen, J. Chem. Phys. 24 (4) (1956) 817-827.

[13] A. Riddiford, Mechanisms for the evolution and ionization of oxygen at platinum electrodes, Electrochim. Acta 4 (1961) 170-178.

[14] F. J. Vidal-Iglesias, J. Solla-Gulln, V. Montiel, A. Aldaz, An easy method for calculating kinetic parameters of electrochemical mechanisms: Temkins formalism, Electrocatalysis 6 (2) (2015) 148-154.

[15] S. Fletcher, Tafel slopes from first principles, J. Solid State Electrochem. 13 (4) (2009) 537-549. 
[16] M. Lefebvre, Establishing the Link Between Multistep Electrochemical Reaction Mechanisms and Experimental Tafel Slopes, Vol. 32 of Modern Aspects of Electrochemistry, 2002, Ch. 3, pp. 249-300.

[17] J.-M. Hu, J.-Q. Zhang, C.-N. Cao, Oxygen evolution reaction on $\mathrm{IrO}_{2}$-based DSA type electrodes: Kinetics analysis of Tafel lines and EIS, Int. J. Hydrogen Energy 29 (8) (2004) 791-797.

[18] E. Gileadi, Problems in interfacial electrochemistry that have been swept under the carpet, J. Solid State Electrochem. 15 (7-8) (2011) 1359-1371.

[19] X. Jiao, C. Batchelor-McAuley, E. Ktelhn, J. Ellison, K. Tschulik, R. G. Compton, The subtleties of the reversible hydrogen evolution reaction arising from the nonunity stoichiometry, J. Phys. Chem. C 119 (17) (2015) 9402-9410.

[20] J. Rossmeisl, A. Logadottir, J. K. Nørskov, Electrolysis of water on (oxidized) metal surfaces, Chem. Phys. 319 (13) (2005) 178-184.

[21] Y.-H. Fang, Z.-P. Liu, Mechanism and Tafel Lines of Electro-Oxidation of Water to Oxygen on $\mathrm{RuO}_{2}(110)$, J. Am. Chem. Soc. 132 (51) (2010) 18214-18222.

[22] M. T. M. Koper, Thermodynamic theory of multi-electron transfer reactions: Implications for electrocatalysis, J. Electroanal. Chem. 660 (2) (2011) 254-260. 


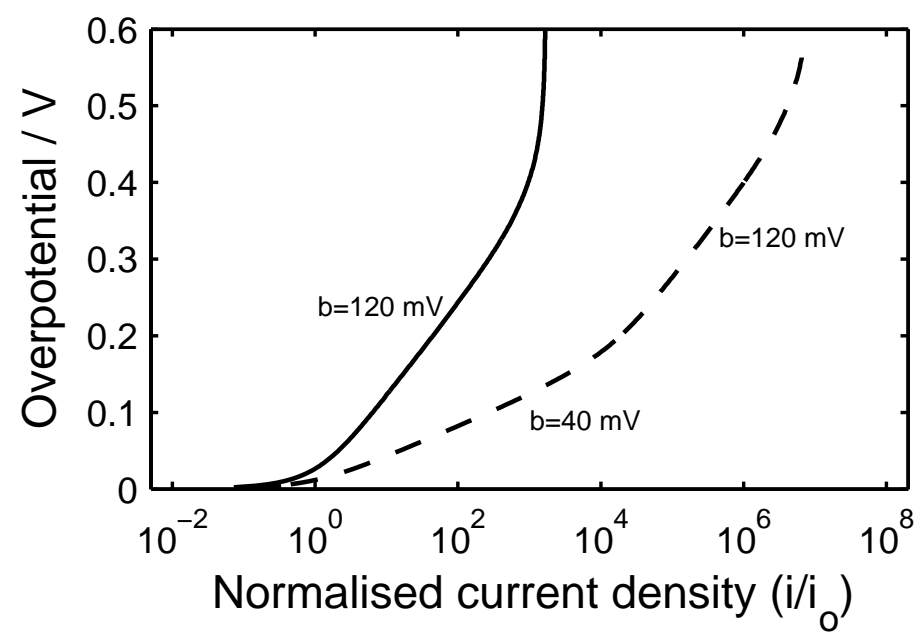

Figure 1: Simulated polarisation curves for the OER. Step 1 limiting (solid line): $K_{2}=10^{0}, K_{3}=10^{-4}$, Step 2 limiting at low potential and step 1 limiting at high overpotential (dashed line): $K_{2}=10^{6}$, $K_{3}=10^{-5}$, Other kinetic parameters used: $k_{1}^{0}=10 \mathrm{~L} \mathrm{~s}^{-1} \mathrm{~mol}^{-1}, k_{2}^{0}=10 \mathrm{~s}^{-1}$ and $k_{3}=100 \mathrm{~s}^{-1}$. Simulated reaction conditions: $\mathrm{T}=298.15 \mathrm{~K}, C_{\mathrm{O}_{2}}=1 \mathrm{bar}, \mathrm{pH}=0$. Note that at very high overpotentials, a limiting current density appears as predicted by Bockris [12]. 


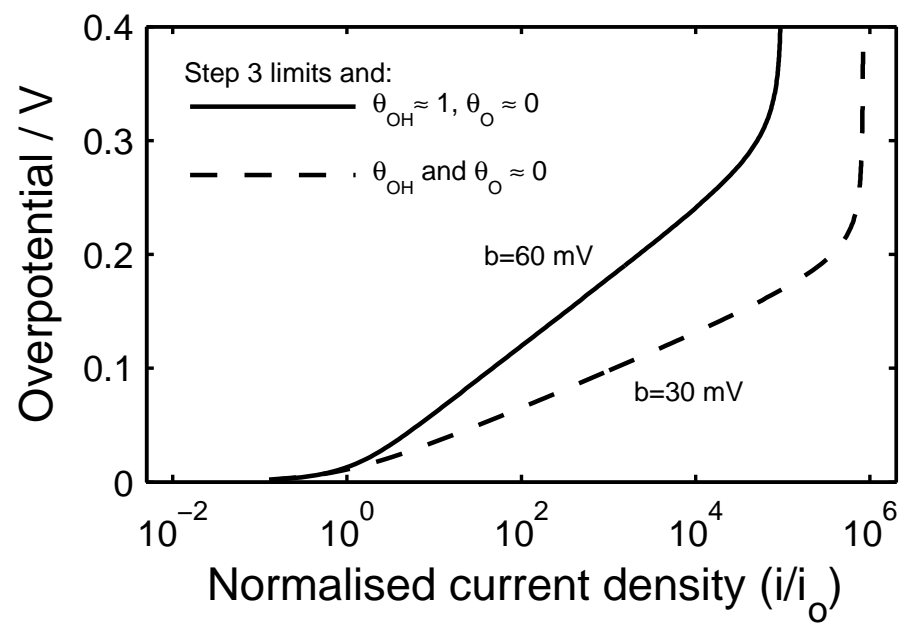

Figure 2: Simulated polarisation curves for the OER when step 3 is the rds. $\theta_{\mathrm{O}} \approx 0$ and $\theta_{\mathrm{OH}} \approx 1$ (solid line): $K^{2}=10^{-5}, K_{3}=10^{2}, \theta_{\mathrm{O}}$ and $\theta_{\mathrm{OH}} \approx 0$ (dashed line): $K_{2}=10^{-2}, K_{3}=10^{2}$, Other parameters are per Figure 1. 

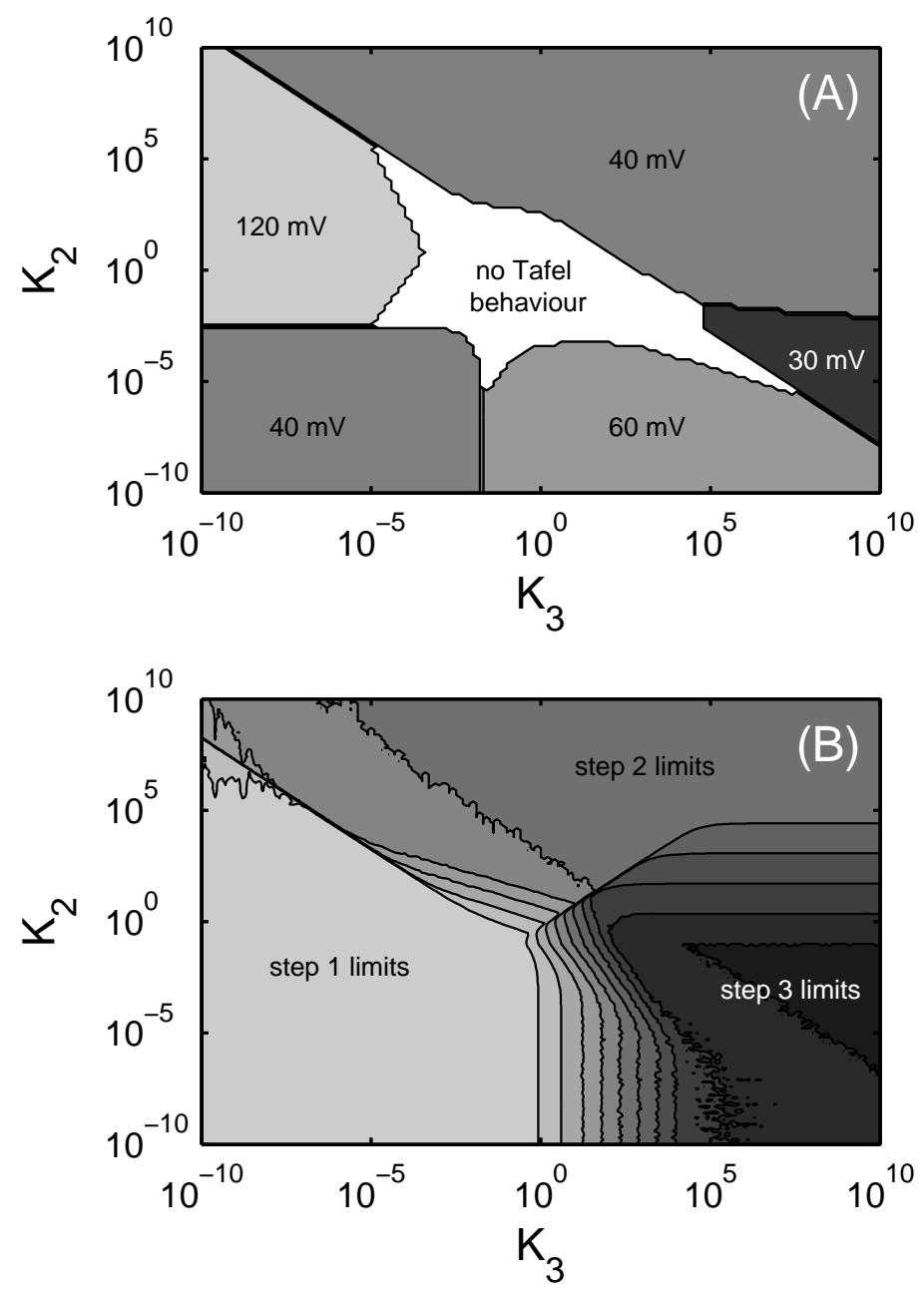

Figure 3: Tafel slopes and rds for the OER following the electrochemical oxide mechanism over a range of $K_{2}$ and $K_{3}$ values. Other parameters are per Figure 1. (A) Tafel slope at low overpotentials, (B) rds 